\title{
Unexpected show up of incomplete fusion at low projectile energies
}

\author{
Pushpendra P. Singh ${ }^{1, \text { a }}$, Abhishek Yadav ${ }^{2}$, Vijay R. Sharma ${ }^{2}$, D. P. Singh ${ }^{2}$, Unnati Gupta ${ }^{2}$, Manoj K. Sharma ${ }^{3}$, R. Kumar $^{4}$, \\ K. S. Golda ${ }^{4}$, R. P. Singh ${ }^{4}$, S. Muralithar ${ }^{4}$, B. P. Singh ${ }^{2}$, R. K. Bhowmik ${ }^{4}$, and R. Prasad ${ }^{4}$ \\ 1 INFN - Laboratori Nazionali di Legnaro, I-35020 Legnaro, Italy \\ 2 Acc. Lab., Department of Physics, A. M. University, Aligarh-202 002, India \\ Physics Department, S. V. College, Aligarh-202 001, India \\ 4 NP-Group, Inter-University Accelerator Center, New Delhi-110 067, India
}

\begin{abstract}
In this paper, some of the important findings of recent measurements performed to study incomplete fusion at low bombarding energies (i.e., $\mathrm{E}_{\text {lab }} \approx 4-7 \mathrm{MeV} /$ nucleon) in ${ }^{12} \mathrm{C},{ }^{16} \mathrm{O}+{ }^{169} \mathrm{Tm}$ systems are briefly summarized. The spin-distributions of xn, pxn, $\alpha \mathrm{xn} / 2 \alpha \mathrm{xn}$ - channels have been measured to probe entirely different $\gamma$-emission patterns (and feeding intensity profiles) during the de-excitation of complete and incomplete fusion objects. Incomplete fusion strength function has been deduced (from the analysis of experimental excitation functions in context of equilibrated compound nucleus decay) to achieve information of onset and strength of incomplete fusion in terms of various entrance channel parameters. Presence of incomplete fusion at slightly above barrier energies has been confirmed by the measurement of linear momentum distribution of heavy recoils. Present results conclusively demonstrate, the existence of incomplete fusion at low bombarding energies, its strong dependence on entrance channel parameters, and the possibility to populate high spin states.
\end{abstract}

\section{Introduction}

The presence of incomplete fusion (ICF $\rightarrow$ where only a part of projectile fuses with target nucleus) at slightly above barrier energies $\left(\mathrm{E}_{l a b} \approx \mathrm{V}_{b}\right)$ and its strong influence on complete fusion $(\mathrm{CF} \rightarrow$ where an excited composite object forms after intimate contact and transient amalgamation of projectile and target nucleus) at relatively higher energies gained resurgent interest in recent years [1-7]. Since the very first experimental observations of ICF [9], the dynamics of ICF has been intensively investigated in terms of: $(i)$ projectile energy, (ii) entrance channel massasymmetry $\left(\mu_{A}=A_{T} / A_{T}+A_{P}\right)$ and/or $Z_{1} \cdot Z_{2}$, (iii) input angular momenta ( $\ell$-values), and ( $i v)$ the shape of interacting partners. Despite a variety of existing experimental and theoretical studies (see ref.[1] for detail), low energy ICF using light heavy ion beams $(\mathrm{A} \leq 20)$ is not fairly understood, thus continues to be an active area of investigations. The most debated and outstanding issues related to ICF at energies $\approx 4-7 \mathrm{AMeV}$ are; $(i)$ the onset and strength of ICF, (ii) the localization of $\ell$-window, and (ii) the usefulness of ICF to populate high-spin states in final reaction products.

For better insights into the aforementioned issues, three sets of experiments have been performed at the InterUniversity Accelerator Center (IUAC), New Delhi, India to probe low energy ICF in ${ }^{12} \mathrm{C},{ }^{16} \mathrm{O}(\approx 4-7 \mathrm{AMeV})+{ }^{169} \mathrm{Tm}$ systems. Detailed discussion on experimental methodologies, obtained results and their interpretations of these

\footnotetext{
a e-mail: pushpendrapsingh@gmail.com, present address: GSI Helmholtz Centre for Heavy Ion Research GmbH, Planckstraße 1, D-64291 Darmstadt, Germany
}

measurements can be found elsewhere $[1,4,7,8]$. However, a brief account of obtained results and their interpretations are discussed in section-2. Some of the important conclusions drawn from these measurements are summarized in the last section of this paper.

\section{Experiments, results and interpretations}

In the first set of experiments, the spin(j)-distributions (SDs) of evaporation residues have been measured using particle- $\gamma$-coincidence technique [1]. However, in the second and third sets of experiments, ICF strength function [4], and linear momentum distribution (LMD) of heavy recoils [7] have been measured using novel activation technique followed by off-line $\gamma$-spectroscopy. Some of the important results obtained from these measurements and their interpretations are given in the following subsections.

\subsection{Spin (J)-distributions: a sensitive probe for ICF}

Aiming to probe the role of high $\ell$-values in the onset of ICF, the SDs of $\mathrm{xn} / \mathrm{pxn} / \alpha-2 \alpha \mathrm{xn}$ - channels have been measured in ${ }^{12} \mathrm{C},{ }^{16} \mathrm{O}+{ }^{169} \mathrm{Tm}$ systems at 10 energies (from $1.02 \mathrm{~V}_{b}$ to $1.64 \mathrm{~V}_{b}$ ) [1]. Particle $(\mathrm{Z}=1,2)-\gamma$ coincidences have been recorded for channel selection. Normalized yield of ERs has been plotted against observed spin $\left(J_{o b s}\right)$ corresponding to the identified $\gamma$-transition to generate SDs. As a representative case, the SDs of $\alpha 4$ n-channel, identified from both Backward $(\mathrm{B} \rightarrow \mathrm{CF})$ and 
EPJ Web of Conferences
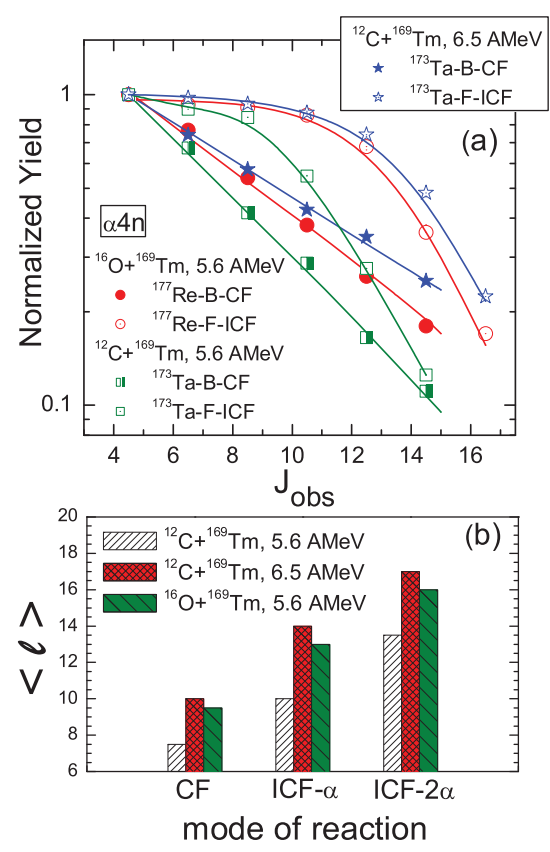

Fig. 1. (a) Experimental SDs for CF- $\alpha 4 \mathrm{n} / \mathrm{ICF}-\alpha 4 \mathrm{n}$ channel. The nomenclature used in this figure indicate the involved reaction dynamics i.e., ' $\mathrm{B}$ ' and ' $\mathrm{F}$ ' respectively indicate $\mathrm{CF}$ and ICF residues identified from backward(B) and forward(F)- $\alpha$-gated spectra. Lines and curves are the result of best fit procedure explained in ref.[1]. (b) The values of mean driving angular momenta $(<\ell>)$ involved in various CF and ICF channels are plotted as a function of reaction modes.

Forward(F $\rightarrow \mathrm{ICF})-\alpha$-gated spectra are shown in Fig.1a. The SDs of $\alpha 4 \mathrm{n}$-channel $(\mathrm{F} \rightarrow \mathrm{ICF})$ are distinctly different than that observed for $\alpha 4 \mathrm{n}$-channel $(\mathrm{B} \rightarrow \mathrm{CF})$. This observation points towards entirely different decay patterns involved in the de-excitation of $\mathrm{CF}$ and ICF objects. The intensity of $\alpha 4 \mathrm{n}$-B-channels (CF) falls off rather quickly with observed spin $\mathrm{J}_{o b s}$, indicating strong feeding and/or broad range spin population during the de-excitation of compound nucleus. For $\alpha 4 \mathrm{n}-\mathrm{F}$-channels(ICF), the intensity appears to be constant up to a certain value of $\mathrm{J}_{o b s}$, and then decreases towards entry side. This indicates the absence of feeding to the lowest members of the 'yrast' band and/or the population of low spin states are strongly hindered in ICF.

Further, the multitude of mean driving angular momenta ( $\ell$-values) involved in different reaction channels have been deduced from the analysis of SDs as explained in ref.[1]. This information is quite useful to examine the possibility to populate high spin states via ICF. The $\ell$ values involved in various modes of reactions are plotted in Fig.1b. As shown in this figure, the $\ell$-values involved in the production of $\mathrm{CF}-\mathrm{xn} / \mathrm{pxn}-\mathrm{B} / \alpha \mathrm{xn}-\mathrm{B}$ and $\mathrm{ICF}-\alpha \mathrm{xn}-\mathrm{F} / 2 \alpha \mathrm{xn}-$ $\mathrm{F}$ channels are found to be $\approx 7.5 \hbar$ and $\approx 10 / 13.5 \hbar$, respectively, at projectile energy $\approx 5.6 \mathrm{AMeV}$. However, at projectile energy $\approx 6.5 \mathrm{AMeV}$, the value of mean driving angular momenta for CF-xn/pxn-B/ $\alpha$ xn-B, and ICF- $\alpha$ xn$\mathrm{F} / 2 \alpha \mathrm{xn}-\mathrm{F}$ channels are found to be $\approx 10 \hbar$ and $\approx 14 / 17 \hbar$, respectively. The enhancement in the value of $\mathrm{J}_{\circ}$ in case of direct- $\alpha$-emitting channels is a direct indication of their origin from higher $\ell$-values as compared to fusion evaporation channels. Apart from that, the $\ell$-values associated with the production of ${ }^{173} \mathrm{Ta}$ via $\mathrm{CF}$ at $\approx 6.5 \mathrm{AMeV}$ (i.e., $\approx$ $9.5 \hbar)$ is achieved via ICF even at lower projectile energy $\approx 5.6 \mathrm{AMeV}$ (i.e., $\approx 10.5 \hbar$ ). Similar characteristics have been observed in case of other reaction channels populated via both CF and ICF. A set of approximate but quite useful correlations emerged from these measurements about the $\ell$-values involved in CF and ICF channels can be written as;

$$
\begin{aligned}
& \text { (i) }{ }^{173} \mathrm{Ta}(\alpha 4 \mathrm{n}), \text { at } \approx 5.6 \mathrm{AMeV} ; \\
& \ell_{(I C F-\alpha 4 n-F)} \approx 1.33 \ell_{(C F-\alpha 4 n-B)}, \\
& \text { (ii) }{ }^{173} \mathrm{Ta}(\alpha 4 \mathrm{n}), \text { at } \approx 6.5 \mathrm{AMeV} ; \\
& \ell_{(I C F-\alpha 4 n-F)} \approx 1.4 \ell_{(C F-\alpha 4 n-B)}, \text { and } \\
& \left(\text { iiii }{ }^{173} \mathrm{Ta}(\alpha 4 \mathrm{n}) ;\right. \\
& \ell_{(I C F-\alpha 4 n)}(5.6 \mathrm{AMeV}) \approx 1.1 \ell_{(C F-\alpha 4 n)}(6.5 \mathrm{AMeV})
\end{aligned}
$$

As mentioned above, ICF can populate ${ }^{173} \mathrm{Ta}(\alpha 4 \mathrm{n})$ at $\approx$ $33 \%$ more angular momentum as compared to that populated via $\mathrm{CF}$ at $\approx 5.6 \mathrm{AMeV}$, and $\approx 40 \%$ more at $\approx 6.5$ $\mathrm{AMeV}$. Further, it is also clear from the (iii)-correlation that, the CF is not able to approach the value of populated angular momenta even at $\approx 6.5 \mathrm{AMeV}$ which has been populated via ICF for the same residue at relatively low bombarding energy, i.e., $\approx 5.6 \mathrm{AMeV}$. This striking feature strongly support the possibility to populate high spin states via ICF even at low bombarding energy.

\subsection{ICF strength function: measure of onset and strength of ICF}

The measurements presented in previous section are relative, and do not give information on how ICF show up with various entrance channel parameters ? For better insights into the influence of ICF on CF, the ICF strength function has been deduced for ${ }^{12} \mathrm{C},{ }^{16} \mathrm{O}+{ }^{169} \mathrm{Tm}$ systems from the analysis of experimental excitation functions in the framework of statistical model code PACE4 [3,4]. Detailed discussion on data reduction procedure and obtained results can be found elsewhere $[3,4]$. As a representative case, the sum of all ICF-channels $\left(\sum \sigma_{I C F}\right)$ is plotted with bombarding energy along with the sum of all CF-channels $\left(\sum \sigma_{C F}\right)$ and total fusion $\left(\sigma_{T F}\right)$ in Fig.2a. The increasing separation between $\Sigma \sigma_{C F}$ and $\sigma_{T F}$ with $\mathrm{E}_{l a b}$ indicates strong energy dependence of ICF. The percentage fraction of ICF $\left(\mathrm{F}_{I C F}\right)$ is plotted with $\mathrm{E}_{l a b}$ in Fig.2b for better visualization of this effect, i.e., termed as ICF strength function. The ICF strength function defines empirical probability of ICF at different projectile energies. The value of $\mathrm{F}_{I C F}$ is found to be $\approx 7 \%$ at $\approx 59 \mathrm{MeV}$, i.e., $1.075 \mathrm{Vb}(7.5 \%$ above the barrier), and increases smoothly up to $\approx 18 \%$ at highest measured energy i.e., $1.64 \mathrm{Vb}$. 
According Morgenstern's systematic [10], ICF contributes significantly above $v_{\text {rel }}=\beta=0.06$ (6\% of c), and the fraction of ICF should increase with entrance channel mass-asymmetry $\left(\mu_{A}\right)$. To test Morgenstern's systematics, the values of $\mathrm{F}_{I C F}$ obtained for ${ }^{12} \mathrm{C},{ }^{16} \mathrm{O}+{ }^{169} \mathrm{Tm}$ systems are plotted with $\beta$ in Fig.3(a). As can be noticed in this figure, the values of $\beta$ are in the range from $\approx 0.027$ $\left(2.7 \%\right.$ of c) to $\approx 0.084\left(8.4 \%\right.$ of c) for ${ }^{12} \mathrm{C}$-beam, and from $\approx 0.014(1.4 \%$ of $\mathrm{c})$ to $\approx 0.053(5.3 \%$ of $\mathrm{c})$ for ${ }^{16} \mathrm{O}$ beam. At given value of $\beta$, no significant ICF contribution is expected. But, the results presented in Fig.3(a) clearly demonstrate the onset of ICF at relatively lower value of $\beta$ i.e, $\approx 0.027\left(\mathrm{~F}_{I C F} \approx 7 \%\right)$ in ${ }^{12} \mathrm{C}+{ }^{169} \mathrm{Tm}$ system, and at $\approx 0.014\left(\mathrm{~F}_{I C F} \approx 10 \%\right)$ in ${ }^{16} \mathrm{O}+{ }^{169} \mathrm{Tm}$ system. In both cases, the observed value of $\mathrm{F}_{I C F}$ is significant even at well below the proposed onset value of $\beta$ (i.e., $6 \%$ of c). Further, the value of $\mathrm{F}_{I C F}$ for ${ }^{12} \mathrm{C}$ is lower than ${ }^{16} \mathrm{O}$-projectile for the entire measured energy range. The difference in $\mathrm{F}_{\text {ICF }}$ for two systems $\left({ }^{12} \mathrm{C},{ }^{16} \mathrm{O}+{ }^{169} \mathrm{Tm}\right)$ can be seen quite clearly, which indicates the dependence of $\mathrm{F}_{I C F}$ on projectile charge and/or on $\mu_{A}$. In order to refine this effect, the values of $\mathrm{F}_{I C F}$ for nearby systems $\left({ }^{12} \mathrm{C}+{ }^{128} \mathrm{Te},{ }^{165} \mathrm{Ho},{ }^{169} \mathrm{Tm}\right.$ and $\left.{ }^{16} \mathrm{O}+{ }^{103} \mathrm{Rh},{ }^{159} \mathrm{~Tb},{ }^{169} \mathrm{Tm}\right)$ are plotted with $\mu_{A}$ in Fig. $3 \mathrm{~b}$ at a constant value of $\beta=0.053$. As shown in this figure, the Morgenstern's systematics do not explain the variation of $\mathrm{F}_{I C F}$ with $\mu_{A}$ for given systems. However, the value of $\mathrm{F}_{I C F}$ increases with $\mu_{A}$ for individual projectiles $\left({ }^{16} \mathrm{O}\right.$ and $\left.{ }^{12} \mathrm{C}\right)$. It is interesting to note that the ${ }^{12} \mathrm{C}+{ }^{169} \mathrm{Tm}$ system is a more mass-asymmetric $\left(\mu_{A}=0.9337\right)$ than ${ }^{16} \mathrm{O}+{ }^{169} \mathrm{Tm}$ system $\left(\mu_{A}=0.9135\right)$, but the value of $\mathrm{F}_{I C F}$ is almost 18 $\%$ higher than that observed for ${ }^{12} \mathrm{C}+{ }^{169} \mathrm{Tm}$ system. The aforementioned observations based on six projectile-target combinations supplement Morgenstern's systematics.

\subsection{Linear momentum distribution of recoils: confirmation of low energy ICF}

Existence of ICF at slightly above barrier energies and the data reduction procedure used to deduce ICF fraction have been validated by the measurement of linear momentum distribution (LMD) of heavy recoils at respective energies $[3,7,8]$. The LMD depends upon the degree of linear momentum transfer $\left(\rho_{L M T}\right)$ from projectile to target nucleus. For ICF, it may be given as: $\rho_{L M T}=\mathrm{P}_{\text {frac }} / \mathbf{P}_{\text {proj } j}$, where $\mathrm{P}_{\text {frac }}$ is the linear momentum of fused fraction of projectile, and $\mathrm{P}_{\text {proj }}$ is the full linear momentum of projectile. As per range-energy calculations, entire LMT (i.e., $\rho_{L M T}$ $=1$ ) from projectile to target nucleus (in case of $\mathrm{CF}$ ) supposed to give maximum recoil velocity to the composite $\left(\mathrm{A}_{\text {target }}+\mathrm{A}_{\text {proj }}\right)$ system, and relatively less recoil velocity to the incompletely fused composite (ICF: $\mathrm{A}_{T}+\mathrm{A}_{\text {proj-frac }}$ ). Consequently, the radio- nuclides populated via small $\rho_{L M T}$ are expected to show relatively smaller recoil penetration depth in the stopping medium as compared to the entire LMT populations. The CF and/or ICF residues have been disentangled from the analysis of LMDs [7].

Fig.4(a) shows the LMD for ${ }^{182} \mathrm{Os}(\mathrm{p} 2 \mathrm{n})$ residue populated in ${ }^{16} \mathrm{O}+{ }^{169} \mathrm{Tm}$ system at $\approx 87 \mathrm{MeV}$. As shown in this figure, the data can be fitted into a Gaussian peak in-
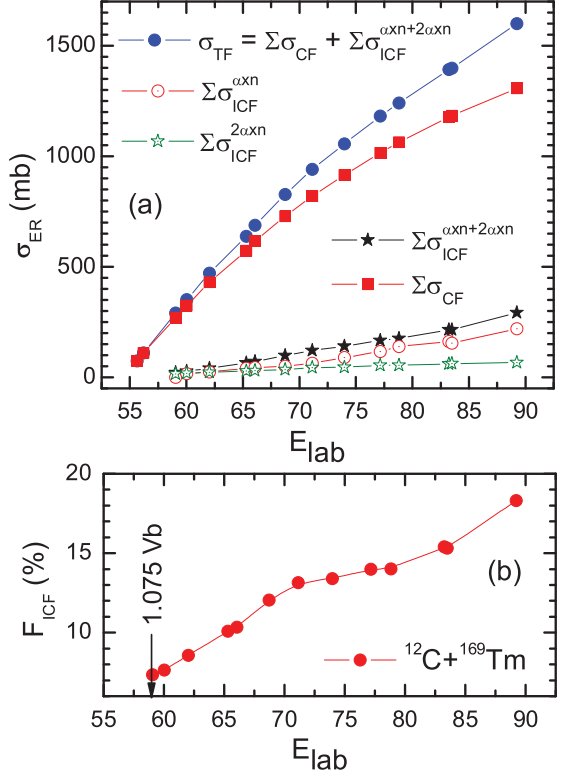

Fig. 2. (a) Total fusion cross-section $\left(\sigma_{T F}=\Sigma \sigma_{C F}+\Sigma \sigma_{I C F}\right)$ along with the sum of all CF-channels $\left(\Sigma_{C F}\right)$ and ICF-channels $\left(\Sigma \sigma_{I C F}\right)$ as a function of projectile energy. $(b)$ the ICF strength function for ${ }^{12} \mathrm{C}+{ }^{169} \mathrm{Tm}$ system deduced from the analysis of experimental EFs. Lines are drawn to guide the eyes.

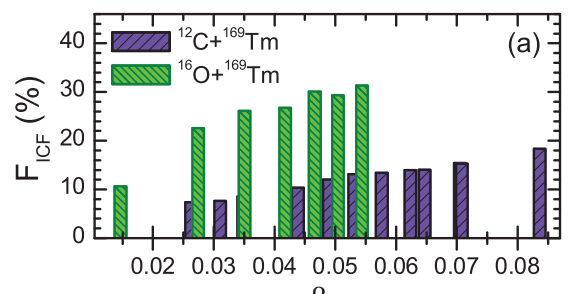

$\beta$

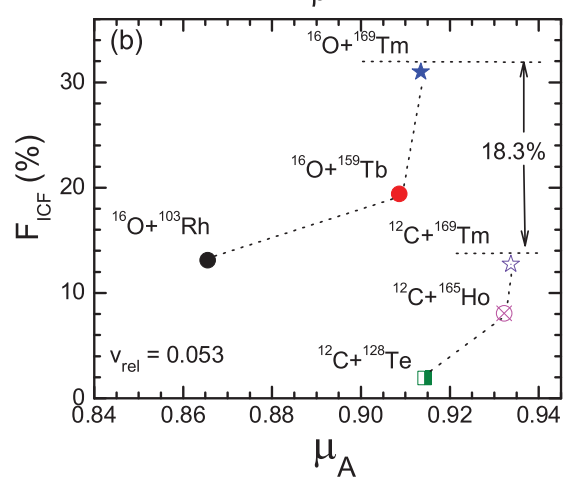

Fig. 3. (a) The value of $\mathrm{F}_{I C F}$ as a function of relative velocity $\left(\mathrm{v}_{\text {rel }}\right)$ for ${ }^{12} \mathrm{C},{ }^{16} \mathrm{O}+{ }^{169} \mathrm{Tm}$ systems. (b) the values of $\mathrm{F}_{\text {ICF }}$ obtained for ${ }^{12} \mathrm{C}+{ }^{128} \mathrm{Te},{ }^{165} \mathrm{Ho},{ }^{169} \mathrm{Tm}$ and ${ }^{16} \mathrm{O}+{ }^{103} \mathrm{Rh},{ }^{159} \mathrm{~Tb},{ }^{169} \mathrm{Tm}$ systems as a function of mass asymmetry $\left(\mu_{A}\right)$ at a constant relative velocity (i.e., $\beta=0.053$ ). The dotted lines drawn through the data points guide the eyes for ${ }^{12} \mathrm{C}$ and ${ }^{16} \mathrm{O}$ projectiles. 

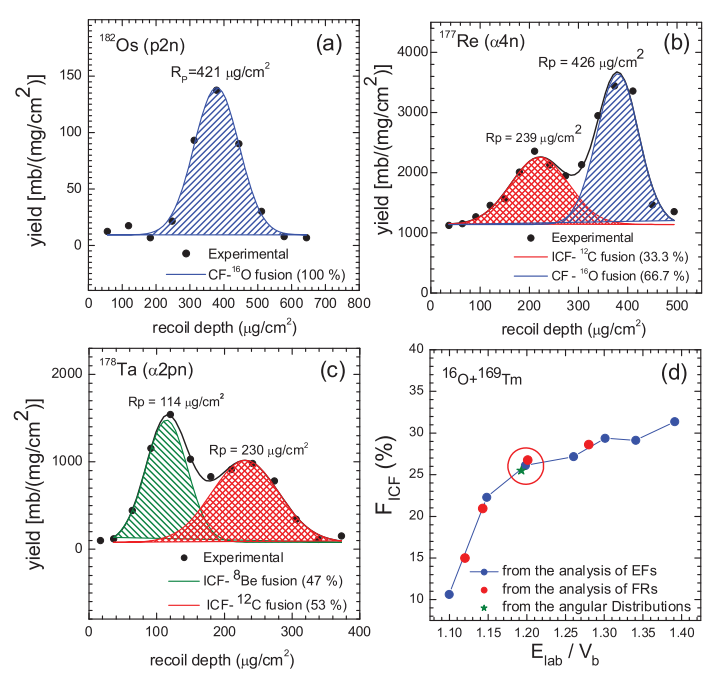

Fig. 4. The experimentally measured linear momentum distributions for $(a){ }^{182} \mathrm{Os}(\mathrm{p} 2 \mathrm{n}),(b){ }^{177} \operatorname{Re}(\alpha 4 \mathrm{n})$, and $(c){ }^{178} \mathrm{Ta}(\alpha 2 \mathrm{pn})$ residues produced in ${ }^{16} \mathrm{O}+{ }^{169} \mathrm{Tm}$ system at $\approx 87 \mathrm{MeV}$. $(d)$ Different approaches to deduce ICF fraction are compared.

dicating single LMT-component involved in the production of this residue. However, Fig.4(b)-(c) show the MD for ${ }^{177} \operatorname{Re}(\alpha 4 \mathrm{n})$ and ${ }^{178} \mathrm{Ta}(\alpha 2 \mathrm{pn})$ residues. As shown in these figures, the LMDs can be resolved into two Gaussian peaks, revealing the presence of more than one LMTcomponents associated with the fusion of ${ }^{16} \mathrm{O},{ }^{12} \mathrm{C}$ and/or ${ }^{8} \mathrm{Be}$ with the target nucleus. As an example, the LMD for ${ }^{177} \operatorname{Re}(\alpha 4 \mathrm{n})$ is showing two LMT components at $\mathrm{R}_{P(\exp )}$ $\approx 426 \mu \mathrm{g} / \mathrm{cm}^{2}$ (due to ${ }^{16} \mathrm{O}$-fusion) and at $\mathrm{R}_{P(\exp )} \approx 239$ $\mu \mathrm{g} / \mathrm{cm}^{2}$ (due to ${ }^{12} \mathrm{C}$-fusion, and an $\alpha$ as spectator) at projectile energy $\approx 87 \mathrm{MeV}$. As such, on the basis of observed $\mathrm{R}_{P(\text { exp })}$, it can be inferred that the residues ${ }^{177} \operatorname{Re}(\alpha 4 \mathrm{n})$ is populated via both CF and ICF processes. This confirms the onset of ICF at these energies. Further, the value of $\mathrm{R}_{P}^{\text {exp }}$ for different residues have also been compared with that estimated by adopting break-up-fusion model given by Udagawa and Tamura [11], and are found to be in good agreement (see refs.[3,7,8]). For the validation of data reduction procedure used to deduce ICF fraction, the values of $\mathrm{F}_{I C F}$ deduced from three different approaches, namely: (i) from the analysis of LMDs, (ii) angular distributions, and (iii) from the analysis of excitation functions, are plotted as a function of $\mathrm{E}_{l a b} / \mathrm{V}_{b}$ in Fig.4(d). As shown in this figure, the values of $\mathrm{F}_{I C F}$ deduced from three different approaches are in good mutual agreement within the experimental uncertainties. The mutual agreement puts faith in our measurements and data reduction procedure.

\section{Summary and conclusions}

This paper briefly summarizes the findings of recent experiments performed to study ICF at energies $\approx 4$ $7 \mathrm{MeV} /$ nucleon in ${ }^{12} \mathrm{C},{ }^{16} \mathrm{O}+{ }^{169} \mathrm{Tm}$ systems. It has been found that ICF significantly contributes to total reaction cross-section even at slightly above barrier energies. The probability of ICF is found to increase with $\mathrm{E}_{l a b}$ and $\mu_{A}$ for individual projectiles. The measurement of LMDs confirms the production of $\alpha$-emitting channels via ICF. As an example, in case of ${ }^{177} \mathrm{Re}$, two LMT components associated with ${ }^{16} \mathrm{O}$ and/or ${ }^{12} \mathrm{C}$-fusion have been observed which indicates the population of this residue via both $\mathrm{CF}$ and ICF. The spin-distributions of ICF- $\alpha \times \mathrm{xn} / 2 \alpha \mathrm{xn}$-channels are found be distinctly different than that observed for $\mathrm{CF}-\mathrm{xn} / \mathrm{pxn} / \alpha \mathrm{xn}$-channels, which indicates entirely different de-excitation patterns in CF and ICF products towards the band head. The population of low spin states are observed to be strongly hindered and/or less fed in ICF, while strong feeding has been observed over the broad spin range in case of CF. The value of mean input angular momentum increases with direct- $\alpha$ multiplicity in forward cone, which indicates the competition from successively opened ICF channels for each $\ell$-value above $\ell_{\text {crit }}$ for normal fusion (CF) even at $\approx 5.6 \mathrm{AMeV}$. This confirms the fact that ICF reactions predominantly occur due to influence of centrifugal potential at higher values of impact parameters, where only CF is expected to be dominant. The comparison of $\ell$-values involved in the production of direct- $\alpha$-emitting and normal- $\alpha$-emitting channels suggests the possibility to populate high spin states in final reaction products via ICF which are not possible to achieve via $\mathrm{CF}$ at a given projectile energy.

We thank Prof. Amit Roy, Director of the IUAC, New Delhi, India for extending experimental facilities to perform these measurements. B.P.S. and R.P. thank to the Department of Science and Technology (D.S.T.) and University Grant Commission (U.G.C.) of India for support.

\section{References}

1. Pushpendra P. Singh et al., Phys. Lett. B671 (2009) 20; Phys. Rev. C80 (2009) 064603, Phys. Rev. C78 (2008) 017602, and the references therein.

2. Alexis Diaz-Torres, J. Phys. G: Nucl. Part. Phys. 37 (2010) 075109, Phys. Rev. Lett. 98 (2007) 152701.

3. Unnati Gupta et al., Nucl. Phys. A 811 (2008) 77-92; Phys. Rev. C80 (2009) 024613.

4. Pushpendra P. Singh et al., Phys. Rev. C 77 (2008) 014607, and the references therein.

5. P. R. S. Gomes et al., Phys. Lett. B601 (2004) 20; Phys. Rev. C73 (2006) 064606.

6. D. J. Hinde and M. Dasgupta, Phys. Rev. C 81 (2010) 064611.

7. Pushpendra P. Singh et al., Euro. Phys. J. A 34 (2007) 29-39, and the references therein.

8. Pushpendra P. Singh et al., Jour. of Phys.: Conf. Series 282 (2011) 012019.

9. H. C. Britt and A. R. Quinton, Phys. Rev. 124 (1961) 877.

10. H. M. Morgenstern et al., Phys. Rev. Lett. 52 (1984) 1104; Phys. Lett. 113B (1982) 463.

11. T. Udagawa and T. Tamura, Phys. Rev. Lett. 45 (1980) 1131. 